\title{
Formulas for the Derivative and Critical Points of Topological Entropy for Anosov and Geodesic Flows ${ }^{\star}$
}

\author{
Anatole Katok ${ }^{1,2}$, Gerhard Knieper ${ }^{3}$ and Howard Weiss ${ }^{1, \star \star}$ \\ 1 Department of Mathematics, Pennsylvania State University, University Park, PA 16802, USA \\ 2 Department of Mathematics, California Institute of Technology, Pasadena, CA 91125, USA \\ 3 Mathematisches Institut, SFB 170, Universität Göttingen, Bunsenstraße 3-5, \\ W-3400 Göttingen, Federal Republic of Germany
}

Received April 3, 1989; in revised form September 17, 1990

\begin{abstract}
This paper represents part of a program to understand the behavior of topological entropy for Anosov and geodesic flows. In this paper, we have two goals. First we obtain some regularity results for $C^{1}$ perturbations. Second, and more importantly, we obtain explicit formulas for the derivative of topological entropy. These formulas allow us to characterize the critical points of topological entropy on the space of negatively curved metrics.
\end{abstract}

\section{Formulation of Results}

The topological entropy, $h_{\mathrm{TOP}}$, measures the exponential growth rate of the number of orbit segments distinguishable with arbitrarily fine but finite precision. In general, it behaves irregularly with respect to perturbations. In the discrete time case, Misiurewicz [Mi1] constructed examples showing that $h_{\text {TOP }}: \operatorname{Diff}^{\infty}\left(M^{n}\right) \rightarrow \mathbb{R}$ is not continuous for $n \geqq 4$, as well as examples showing that $h_{\text {TOP }}$ is not uppersemicontinuous in the $C^{k}$ topology for $k<\infty$ in every dimension $n \geqq 2$ [Mi2]. Here Diff ${ }^{\infty}\left(M^{n}\right)$ denotes the space of $C^{\infty}$ diffeomorphisms on a compact $n$ dimensional manifold equipped with the $C^{\infty}$ topology. Yomdin [Y] and Newhouse [N] proved that $h_{\text {TOP }}: \operatorname{Diff}^{\infty}\left(M^{n}\right) \rightarrow \mathbb{R}$ is upper semicontinuous. For $n=2$, Katok [K3] proved lower semicontinuity. By combining these two results, one sees that $h_{\text {TOP }}:$ Diff ${ }^{\infty}\left(M^{2}\right) \rightarrow \mathbb{R}$ is continuous. This result also holds for $C^{\infty}$ flows on three dimensional manifolds.

The structural stability of Anosov diffeomorphisms [A] implies that $h_{\text {TOP }}$ is locally constant. For Anosov flows, the structural stability [A] involves a time

\footnotetext{
* Partially supported by NSF grant DMS-8514630

$\star \star$ Chaim Weizmann Research Fellow and NSF Postdoctoral Research Fellow
} 
change and the topological entropy may change. However, the structural stability implies that $h_{\text {TOP }}$ is continuous. Misiurewicz [Mi2] has shown that for general $C^{k}$ flows on $M^{n}, k<\infty$ and $n \geqq 3, h_{\text {TOP }}$ need not be continuous.

This paper represents part of a program to understand the behavior of topological entropy for Anosov and geodesic flows. The principal regularity results were obtained in [KKPW]. Later, related results were obtained by Contreras [C]. In this paper, we have two goals. First we obtain some regularity results for $C^{1}$ perturbations (Theorems 1 and 2). Second and more importantly, we obtain explicit formulas for the derivative of topological entropy. These formulas allow us to characterize the critical points of topological entropy on the space of negatively curved metrics.

Our first result is an almost straightforward consequence of the results by Manning $[\mathrm{M}]$ and Freire and Mañé [FM].

Theorem 1. Let $\left(M^{n}, g\right)$ be a compact $n$-dimensional $C^{2}$ Riemannian manifold without conjugate points and let $g_{\lambda},-\varepsilon \leqq \lambda \leqq \varepsilon$, be a $C^{1}$ perturbation through metrics without conjugate points. If $\phi_{\lambda}$ is the geodesic flow associated to $g_{\lambda}$, then

$$
\lambda \rightarrow h_{T}\left(g_{\lambda}\right):=h_{\text {TOP }}\left(\phi_{\lambda}\right)
$$

is a Lipschitz function.

The next two theorems contain the principle results of this paper:

Theorem 2. Let $\phi^{t}$ be a $C^{1}$ Anosov flow on a compact $n$-dimensional manifold $X$ and let $\phi_{\lambda}^{t},-\varepsilon \leqq \lambda \leqq \varepsilon$ be a $C^{1}$ perturbation of $\phi^{t}=\phi_{0}^{t}$. Then $h_{T}(\lambda)=h_{\mathrm{TOP}}\left(\phi_{\lambda}\right)$ is a $C^{1}$ function and the derivative is given by

$$
\left.\frac{\partial}{\partial \lambda}\right|_{\lambda=\lambda_{0}} h_{T}(\lambda)=\left.h_{T}(0) \cdot \int_{X} \frac{\partial}{\partial \lambda}\right|_{\lambda=\lambda_{0}} a_{\lambda}(p) d \mu_{0},
$$

where $\mu_{0}$ denotes the Margulis measure (the unique measure of maximal entropy for $\phi^{t}[\mathrm{Ma} 2]$ ) with respect to the unperturbed Anosov flow and the function $a_{\lambda}(p)$ compares the infinitesimal generator of $\phi_{\lambda}$ to the infinitesimal generator of $\phi$. One can think of $a_{\lambda}(p)$ as an infinitesimal time change.

Theorem 3. Let $(M, g)$ be a compact $C^{2}$ Riemannian manifold of negative sectional curvature and let $g_{\lambda},-\varepsilon \leqq \lambda \leqq \varepsilon$, be a $C^{2}$ perturbation of $g=g_{0}$. If $\phi_{\lambda}$ is the geodesic flow associated to $g_{\lambda}$, then $h_{T}\left(g_{\lambda}\right)=h_{\mathrm{TOP}}\left(\phi_{\lambda}\right)$ is a $C^{1}$ function, and the derivative is given by

$$
\left.\frac{\partial}{\partial \hat{\lambda}}\right|_{\lambda=\lambda_{0}} h_{T}\left(g_{\lambda}\right)=-\left.\frac{h_{T}\left(g_{0}\right)}{2} \cdot \int_{S M} \frac{\partial}{\partial \hat{\lambda}}\right|_{\lambda=\lambda_{0}} g_{\lambda}(v, v) d \mu_{g_{0}},
$$

where $\mu_{g_{0}}$ denotes the Margulis measure of $g_{0}$.

Remarks.

a) Since the geodesic flow on a negatively curved manifold is Anosov [A], Theorem 2 also applies in this case. The two formulas are different. Furthermore, the proof of Theorem 3 does not use structural stability.

b) In $[\mathrm{KKPW}]$ the authors prove, among other regularity results, that for a $C^{2}$ 
perturbation of a $C^{1}$ Anosov flow, $h_{\text {Top }}$ is $C^{1}$. However, they do not obtain a formula for the derivative.

c) Theorem 3 can be extended to Rank 1 manifolds of nonpositive curvature provided one can ensure the existence of a unique measure of maximal entropy. This was established by Ursula Hamenstadt (unpublished). Once the theorem is known for Rank 1 manifolds, it follows from the classification of compact manifolds of nonpositive curvature and the rigidity of higher rank manifolds (see Appendix 1 of [BGS] for a convenient summary) that the theorem is valid for arbitrary manifolds of nonpositive curvature.

The following result contains an interesting application of the formula from Theorem 3:

Theorem 4. Let $M$ be a compact surface and let $\mathfrak{R}(M)$ denote the submanifold of negatively curved $C^{2}$ metrics on $M$ having area equal to 1 . Then $h_{T}: \Re(M) \rightarrow \mathbb{R}$ has a critical point at $g_{0} \in \mathfrak{R}(M)$ if and only if $g_{0}$ is a metric of constant negative curvature.

We would like to point out several open questions related to our results.

Conjecture 1 . Let $\mathfrak{R}(M)$ denote the submanifold of negatively curved $C^{2}$ metrics on a compact manifold $M$ having volume equal to 1 . Then $h_{T}: \mathfrak{R}(M) \rightarrow \mathbb{R}$ has a critical point at $g_{0}$ if and only if the Margulis measure and the Liouville measure of the geodesic flow for $g_{0}$ coincide.

The next conjecture would follow from Conjecture 1 and a well-known entropy rigidity conjecture of the first author $[\mathrm{K} 2, \mathrm{BK}]$.

Conjecture 2. Let $\mathfrak{R}(M)$ denote the submanifold of negatively curved $C^{2}$ metrics on a compact manifold $M$ having volume equal to 1 . Then $h_{T}: \mathfrak{R}(M) \rightarrow \mathbb{R}$ has critical point at $g_{0}$ if and only if $g_{0}$ is locally symmetric.

Our last conjecture would imply Conjecture 1 even if it is established only under the extra assumption that $\mu$ is the Margulis measure (cf. proof of Theorem 4 below).

Conjecture 3. Let $(M, g)$ be a compact Riemannian manifold of negative sectional curvature. Suppose $\mu$ is a Borel probability measure on SM which is invariant under the geodesic flow, and projects to Lebesgue measure on $M$, i.e., $\mu$ and the Liouville measure coincide on $\pi^{-1}(B(M)):=\left\{\pi^{-1}(A) \mid A \in B(M)\right\}$, where $B(M)$ is the $\sigma$-algebra of Borel subsets on $M$. Then $\mu$ is the Liouville measure.

\section{Topological Entropy for Geodesic Flows on Manifolds Without Conjugate Points}

Let $(M, g)$ be a compact Riemannian manifold and $\tilde{M}$ the universal covering of $M$. For every $p \in \tilde{M}$, Manning $[\mathrm{M}]$ showed that the limit

$$
\lim _{r \rightarrow \infty} \frac{\log \operatorname{vol}_{g} B_{g}(p, r)}{r}=\Lambda_{g}
$$

exists and is independent of $p$. Here $B_{g}(p, r)$ denotes the geodesic ball of radius $r>0$ in the universal covering $\tilde{M}$ and $\operatorname{vol}_{g}$ is the volume associated to $g$. Margulis 
[Ma2], for metrics of negative curvature, Manning [M], for metrics with no focal points, and Freire and Mañé [FM], for metrics without conjugate points, showed that $\Lambda_{g}$ coincides with the topological entropy $h_{T}(g)$ of the geodesic flow in the compact quotient.

Lemma 1. Let $(M, g)$ be a compact $n$-dimensional Riemannian manifold and let $\left\{g_{\lambda}\right\},-\varepsilon<\lambda<\varepsilon$ be a $C^{1}$ perturbation of $g=g_{0}$. Then there are Lipschitz functions $M(\hat{\lambda})$ and $m(\hat{\lambda}), M(0)=m(0)=1$ such that: a) For all $p, q \in M, m(\lambda) \leqq \frac{d_{\lambda}(p, q)}{d_{0}(p, q)} \leqq M(\lambda)$, where $d_{\lambda}$ denotes the distance generated
by $g_{\lambda}$.

b) For all measurable sets $S \subset M$

$$
m(\lambda)^{n} \leqq \frac{\operatorname{vol}_{\lambda}(S)}{\operatorname{vol}_{0}(S)} \leqq M(\lambda)^{n},
$$

where $\operatorname{vol}_{\lambda}$ denotes the volume generated by $g_{\lambda}$.

Proof. Define

$$
M(\lambda)=\max _{v \in(S M)_{0}}\|v\|_{\lambda}, \quad m(\lambda)=\min _{v \in(S M)_{0}}\|v\|_{\lambda},
$$

where $(S M)_{0}$ is the unit tangent bundle of $M$ with respect to $g_{0}$, and $\|v\|_{\lambda}$ is the norm of $v$ in the $g_{\lambda}$ metric. From this definition, the inequality in (a) is evident.

Comparing the eigenvalues of $g_{\lambda}$ and $g_{0}$ one obtains:

$$
m(\lambda)^{n} \leqq \sqrt{\frac{\operatorname{det} g_{\lambda}}{\operatorname{det} g_{0}}} \leqq M(\lambda)^{n}
$$

This immediately impiies (b).

To see that $M(\lambda)$ is Lipschitz we observe that (1) $\|v\|_{0}=1$ for all $v \in(S M)_{0}$ and (2) $D=\sup _{\lambda} \sup _{v \in(S M)_{0}} \frac{\partial}{\partial \lambda}\|v\|_{\lambda}<\infty$. Since $\|v\|_{\lambda}$ is a $C^{1}$ function on $(S M)_{0} \times(-\varepsilon, \varepsilon),(1)$ and (2) imply that $|M(\lambda)-M(0)| \leqq D \cdot \lambda$. The same argument applies to $m(\lambda)$.

Remark. The inequalities (a) and (b) remain valid if we replace $M$ by a covering space equipped with the lifted metric.

Proof of Theorem 1. Let $\tilde{M}$ be the universal cover of $M$ and fix $p_{0} \in \tilde{M}$. Then part (a) of Lemma 1 implies:

$$
B_{g_{0}}\left(p_{0}, \frac{r}{M(\lambda)}\right) \subseteq B_{g_{\lambda}}\left(p_{0}, r\right) \subseteq B_{g_{0}}\left(p_{0}, \frac{r}{m(\lambda)}\right)
$$

Hence,

$$
\operatorname{vol}_{g_{0}}\left(B_{g_{0}}\left(p_{0}, \frac{r}{M(\lambda)}\right)\right) \leqq \operatorname{vol}_{g_{0}}\left(B_{g_{\lambda}}\left(p_{0}, r\right)\right) \leqq \operatorname{vol}_{g_{0}}\left(B_{g_{0}}\left(p_{0}, \frac{r}{m(\lambda)}\right)\right) .
$$

Therefore,

$$
\frac{1}{M(\lambda)} \cdot h_{T}\left(g_{0}\right) \leqq \lim _{r \rightarrow \infty} \frac{\log \operatorname{vol}_{g_{0}} B_{g_{\lambda}}\left(p_{0}, r\right)}{r} \leqq \frac{1}{m(\lambda)} \cdot h_{T}\left(g_{0}\right)
$$


Part (b) of Lemma 1 implies:

$$
\lim _{r \rightarrow \infty} \frac{\log \operatorname{vol}_{g_{0}} B_{g_{\lambda}}\left(p_{0}, r\right)}{r}=\lim _{r \rightarrow \infty} \frac{\log \operatorname{vol}_{g_{\lambda}} B_{g_{\lambda}}\left(p_{0} r\right)}{r}=h_{T}\left(g_{\lambda}\right) .
$$

\section{Topological Entropy for Anosov Flows}

Let $\phi$ be an Anosov flow on a compact space $X$. Margulis [Ma1] and Bowen [B] have shown that the topological entropy $h_{T}$ is obtained by:

$$
h_{T}(\phi)=\lim _{t \rightarrow \infty} \frac{\log \# P(t)}{t},
$$

where $P(t)$ denotes the set of periodic orbits of (prime) period $\leqq t$. To prove Theorem 2 , it is important to count only those closed orbits which are almost uniformly distributed with respect to the Margulis measure. Given $\varepsilon>0$ and a continuous function $f$, we say that a closed orbit $\left\{\phi^{s} v\right\}_{s=0}^{l}$ of period $l$ is $(\varepsilon, f)$ uniformly distributed with respect to a measure $\mu$ if

$$
\left|\frac{1}{l} \int_{0}^{l} f\left(\phi^{t} v\right) d t-\int_{X} f d \mu\right|<\varepsilon
$$

Proposition 2. Let $\phi$ be an Anosov flow, $\mu$ be an invariant ergodic measure for $\phi$, and $f$ a continuous function on $X$. For every $\varepsilon>0$ let $P_{\mu}^{\varepsilon, f}(t)$ be the set of $(\varepsilon, f)$ uniformly distributed closed orbits of period $\leqq t$. Then:

$$
\liminf _{t \rightarrow \infty} \frac{\log \# P_{\mu}^{\varepsilon, f}(t)}{t} \geqq h_{\mu}(\phi)
$$

where $h_{\mu}(\phi)$ is the measure theoretic entropy of $\phi$ with respect to $\mu$.

Proof. The proof follows from the methods which the first author developed in [K1] and [K2].

Remarks.

a) The proposition remains true for any flow $\phi$ if $\mu$ is an invariant ergodic measure with non-zero Lyapunov exponents.

b) If $\mu$ is the Margulis measure of $\phi$ then:

$$
\lim _{t \rightarrow \infty} \frac{\log \# P_{\mu}^{\varepsilon, f}(t)}{t}=h_{T}(\phi)
$$

The next ingredient for the proof of Theorem 2 is a refined version of the structural stability of Anosov flows.

Proposition 3. (Structural Stability for Anosov Flows) Let $\Phi$ be a $C^{1}$ vector field on a compact manifold $X$ which generates an Anosov flow $\phi^{t}$. Define

$$
C_{\Phi}^{0}(X)=\left\{u: X \rightarrow X \mid u \text { homeomorphism such that }\left.\frac{d}{d t}\right|_{t=0} u \circ \phi^{t}=: D_{\Phi} u \text { exists }\right\},
$$

and let $C^{0}(X, \mathbb{R})$ be the space of continuous functions on $X$. Then there exists a $C^{1}$ 
neighborhood $U(\Phi)$ of $\Phi$ and a $C^{1}$ map

$$
\begin{aligned}
S: U(\Phi) & \rightarrow C_{\Phi}^{0}(X) \times C^{0}(X, \mathbb{R}) \\
\psi & \rightarrow(u, \gamma)
\end{aligned}
$$

which solves the structural stability equation

$$
\Psi \circ u=\gamma \cdot D_{\Phi} u .
$$

Corollary 4. (Dependence on Parameters) Let $\Phi$ be as in Proposition 3 and let $\Phi_{\lambda}$ be a $C^{1}$ perturbation of $\Phi=\Phi_{0}$. Then there exists $\varepsilon>0$ such that:

a) For each fixed $p_{0} \in X$,

$$
\lambda \rightarrow u_{\lambda}\left(p_{0}\right), \quad \lambda \rightarrow \gamma_{\lambda}\left(p_{0}\right) \text { are } C^{1} \text { for } \lambda \in(-\varepsilon,+\varepsilon) .
$$

b) For each fixed $\lambda_{0} \in(-\varepsilon, \varepsilon)$,

$$
p \rightarrow u_{\lambda_{0}}(p), \quad p \rightarrow \gamma_{\lambda_{0}}(p)
$$

and

$$
p \rightarrow u_{\lambda}(p),\left.\quad p \rightarrow \frac{\partial}{\partial \lambda}\right|_{\lambda=\lambda_{0}} \gamma_{\lambda}(p)
$$

are continuous functions on $X$.

Proof of Corollary 4. Since $S$ is $C^{1}$ we obtain: For each fixed $p_{0}, \lambda \rightarrow S\left(\phi_{\lambda}\right)\left(p_{0}\right)$ is a $C^{1}$ function, and for each fixed $\lambda_{0}$ and $j \in\{0,1\},\left.p \rightarrow \frac{\partial^{j}}{\partial \lambda^{j}}\right|_{\lambda=\lambda_{0}} S\left(\phi_{\lambda}\right)(p)$ is continuous
on $X$.

We present a sketch of the proof of Proposition 3 in the appendix. Also see the appendix to [LMM].

The following formulas for the time change in structural stability are obtained by integrating the infinitesimal time change.

Lemma 5. Let the vector field $\Psi$ be $C^{1}$ close to the Anosov vector field $\Phi$ and let $(u, \gamma) \in C_{\Phi}^{\alpha}(X) \times C^{\alpha}(X, \mathbb{R})$ denote the solution to the structural stability equation

$$
\Psi \circ u=\gamma \cdot D_{\Phi} u .
$$

If $\phi^{t}$ and $\psi^{s}$ are the flows generated by $\Phi$ and $\Psi$, then the time change equations

have the solutions

$$
\begin{aligned}
\psi^{s(t, p)} u(p) & =u^{\circ} \phi^{t}(p), \\
\psi^{s} u(p) & =u^{\circ} \phi^{t(s, p)}(p)
\end{aligned}
$$

$$
\begin{aligned}
s(t, p) & =\int_{0}^{t} \frac{1}{\gamma\left(\phi^{x}(p)\right)} d x, \\
t\left(s, u^{-1}(p)\right) & =\int_{0}^{s} \gamma\left(u^{-1} \phi^{x}(p)\right) d x .
\end{aligned}
$$

Proof. Simple calculation.

The following inequality is the key step in the proof of Theorem 2 (cf. the proof of Theorem 1 in [K4]). 
Proposition 6. Let $\phi$ be a $C^{1}$ Anosov flow and $\psi$ be a perturbation $C^{1}$ close to $\phi$. Then

$$
\frac{h_{T}(\phi)}{\int_{X} \frac{1}{\gamma} d \mu_{\phi}} \leqq h_{T}(\psi) \leqq h_{T}(\phi) \cdot \int_{X} \gamma \circ u^{-1} d \mu_{\psi},
$$

where $\mu_{\phi}$ and $\mu_{\psi}$ are the Margulis measures with respect to $\phi$ and $\psi, u$ is a conjugating homeomorphism, and $\gamma$ is the corresponding infinitesimal time change.

Proof. Given $\varepsilon>0$, let $P_{\phi}^{\varepsilon, \gamma^{-1}}(t)$ be the set of closed orbits of $\phi$ of period $\leqq t$, which are $\left(\varepsilon, \gamma^{-1}\right)$ uniformly distributed with respect to the Margulis measure $\mu_{\phi}$.

Let $p$ be a periodic point for $\phi$ with period $l_{\phi}(p)$ whose orbit $\sigma$ belongs to the set $P_{\phi}^{\varepsilon, \gamma^{-1}}(t)$. The period of the perturbed orbit $\psi^{s}(u(p))$ is given by $s\left(l_{\phi}(p), p\right)$. Because $\sigma$ is $\left(\varepsilon, \gamma^{-1}\right)$ uniformly distributed, it follows from Lemma 5 that:

$$
\frac{s\left(l_{\phi}(p), p\right)}{l_{\phi}(p)} \leqq \varepsilon+\int_{X} \frac{1}{\gamma} d \mu_{\phi}=: a(\varepsilon)
$$

Using Proposition 2, we obtain:

$$
h_{T}(\phi)=\lim _{t \rightarrow \infty} \frac{\log \# P_{\phi}^{\varepsilon, \gamma^{-1}}(t)}{t} \leqq a(\varepsilon) \cdot \lim _{t \rightarrow \infty} \frac{\log \# P_{\psi}(t \cdot a(\varepsilon))}{t \cdot a(\varepsilon)}=a(\varepsilon) h_{T}(\psi) .
$$

Since $\varepsilon>0$ is arbitrary, we conclude that

$$
h_{T}(\phi) \leqq h_{T}(\psi) \cdot \int_{X} \frac{1}{\gamma} d \mu_{\phi}
$$

Similarly, using the second assertion of Lemma 5, we derive:

$$
h_{T}(\psi) \leqq h_{T}(\phi) \int_{X} \gamma \circ u^{-1} d \mu_{\psi} .
$$

Proof of Theorem 2. Let $\phi_{\lambda}$ be a $C^{1}$ perturbation of $\phi=\phi_{0},-\varepsilon \leqq \lambda \leqq \varepsilon$.

Proposition 6 implies that:

$$
\begin{aligned}
h_{T}\left(\phi_{0}\right)\left(\frac{1}{\int_{X} \frac{1}{\gamma_{\lambda}} d \mu_{0}}-1\right) & \leqq h_{T}\left(\phi_{\lambda}\right)-h_{T}\left(\phi_{0}\right) \\
& \leqq h_{T}\left(\phi_{0}\right)\left(\int_{X} \gamma_{\lambda}{ }^{\circ} u_{\lambda}^{-1} d \mu_{\lambda}-1\right),
\end{aligned}
$$

where $\gamma_{\lambda}, u_{\lambda}$ are as in Proposition 6 but now depend on the parameter $\lambda$, and $\mu_{\lambda}$ is the Margulis measure for $\phi_{\lambda}$. Linearizing in $\lambda$ at $\lambda=0$ leads to:

$$
\frac{1}{\gamma_{\lambda}(p)}=1-\left.\lambda \cdot \frac{\partial}{\partial s}\right|_{s=0} \gamma_{s}(p)+o(\lambda)
$$

and

$$
\gamma_{\lambda}\left(u_{\lambda}^{-1}(p)\right)=1+\left.\lambda \cdot \frac{\partial}{\partial s}\right|_{s=0} \gamma_{s}\left(u_{\lambda}^{-1}(p)\right)+o(\lambda)
$$


Since $\left|\frac{\partial}{\partial \lambda} u_{\lambda}^{-1}(p)\right| \leqq$ constant for all $p \in X$ and $\left.\frac{\partial}{\partial s}\right|_{s=0} \gamma_{s}$ is uniformly continuous, we obtain:

$$
\gamma_{\lambda}\left(u_{\lambda}^{-1}(p)\right)=1+\left.\lambda \cdot \frac{\partial}{\partial s}\right|_{s=0} \gamma_{s}(p)+\lambda \cdot \varepsilon(\lambda)+o(\lambda),
$$

where $\varepsilon(\lambda) \rightarrow 0$ as $\lambda \rightarrow 0$. Substituting this in the equation above yields:

$$
\begin{aligned}
& h_{T}\left(\phi_{0}\right)\left(\lambda \cdot \int_{X}\left(\left.\frac{\partial}{\partial s}\right|_{s=0} \gamma_{s}\right) d \mu_{0}+o_{1}(\lambda)\right) \leqq h_{T}\left(\phi_{\lambda}\right)-h_{T}\left(\phi_{0}\right) \\
& \leqq h_{T}\left(\phi_{0}\right)\left(\lambda \cdot \int_{X}\left(\left.\frac{\partial}{\partial s}\right|_{s=0} \gamma_{s}\right) d \mu_{\lambda}+o_{2}(\lambda)\right),
\end{aligned}
$$

for suitable $o_{1}(\hat{\lambda})$ and $o_{2}(\lambda)$.

We remark that this inequality already implies Lipschitz continuity for $\lambda \rightarrow h_{T}\left(\phi_{\lambda}\right)$. The proof of Theorem 2 follows from the next lemma.

Lemma 7. (Weak Continuity of the Margulis Measure) Let $\phi_{n}$ be a sequence of Anosov flows, $\phi_{n} \stackrel{C^{1}}{\longrightarrow} \phi$ and $\mu_{n}, \mu$ be the associated Margulis measures. Then $\mu_{n}$ converges to $\mu$ in the weak topology.

Proof. Let $\mu^{*}$ be any weak limit of $\mu_{n}$. We show that $\mu^{*}=\mu$. Since $\phi$ is expansive, $\mu \rightarrow h_{\mu}(\phi)$ is upper semicontinuous [W]. This, along with the variational principle, implies that:

$$
h_{T}(\phi) \geqq h_{\mu^{*}}(\phi) \geqq \limsup _{n_{k} \rightarrow \infty} h_{\mu_{n_{k}}}\left(\phi_{n_{k}}\right) .
$$

The structural stability of Anosov flows immediately implies that topological entropy is continuous. Hence,

$$
\underset{n_{k} \rightarrow \infty}{\limsup } h_{\mu_{n_{k}}}\left(\phi_{n_{n}}\right)=\limsup _{n_{k} \rightarrow \infty} h_{T}\left(\phi_{n_{k}}\right)=h_{T}(\phi) .
$$

This implies that $h_{T}(\phi)=h_{\mu^{*}}(\phi)$, and since the measure of maximal entropy is unique, $\mu^{*}=\mu$.

\section{Topological Entropy for Geodesic Flows on Manifolds of Negative Curvature}

The following inequality is a counterpart of the one in Proposition 6. Its proof is even closer to the proof of Theorem 1 in [K4].

Proposition 8. Let $\left(M, g_{0}\right)$ be a compact manifold of negative curvature and $g$ be another metric of negative curvature on $M$. Then:

$$
\frac{h_{T}\left(g_{0}\right)}{\int_{(S M)_{q_{0}}}\|v\|_{g} d \mu_{g_{0}}} \leqq h_{T}(g) \leqq h_{T}\left(g_{0}\right) \cdot \int_{(S M)_{q}}\|v\|_{g_{0}} d \mu_{g},
$$

where $\mu_{g_{0}}$ and $\mu_{g}$ are the Margulis measures associated to $g_{0}$ and $g$.

Proof. Again we use the fact that the topological entropy is given by the exponential 
growth rate of the number of closed geodesics, i.e.

$$
h_{T}(g)=\lim _{t \rightarrow \infty} \frac{\log \# P_{g}(t)}{t}
$$

where $P_{g}(t)$ denotes the set of closed geodesics of (prime) period $\leqq t$ in the metric $g$. Let $\Pi(M)$ be the set of free homotopy classes of closed curves in $M$. Given a metric $g$ of negative curvature on $M$, we associate to each $\alpha \in \Pi(M)$ the uniquely determined closed geodesic $c_{\alpha, g} \in \alpha$. If $L_{g}: \Pi(M) \rightarrow \mathbb{R}$ is the length function with respect to the metric $g$, then $c_{\alpha, g}$ is characterized by

$$
L_{g}\left(c_{\alpha, g}\right)=\inf _{c \in \alpha} L_{g}(c) \text {. }
$$

Choose $\varepsilon>0$ arbitrary. As before let $P_{g}^{\varepsilon, \|} \|_{g_{0}}(t)$ be the set of all closed geodesics in the metric $g$ of period $\leqq t$ which are $\left(\varepsilon,\|\|_{g_{0}}\right)$ uniformly distributed with respect to the Margulis measure $\mu_{g}$. If $c_{\alpha, g} \in P_{g}^{\varepsilon, \|} \|_{g_{0}}(t)$ is parametrized by arclength, we obtain the following for the period of the corresponding closed geodesics $c_{\alpha, g_{0}}$,

$$
\begin{aligned}
L_{g_{0}}\left(c_{\alpha, g_{0}}\right) & \leqq L_{g 0}\left(c_{\alpha, g}\right)=\int_{0}^{L_{g}\left(c_{\alpha, g}\right)}\left\|\dot{c}_{\alpha, g}(s)\right\|_{g_{0}} d s \\
& \leqq L_{g}\left(c_{\alpha, g}\right)\left(\varepsilon+\int_{(S M)_{g}}\|v\|_{g_{0}} d \mu_{g}\right)=: L_{g}\left(c_{\alpha, g}\right) \cdot a(\varepsilon) .
\end{aligned}
$$

Applying Proposition 2 yields:

$$
\begin{aligned}
h_{T}(g) & =\lim _{t \rightarrow \infty} \frac{\log \# P_{g}^{\varepsilon, \|} \|_{g_{0}}(t)}{t} \\
& \leqq a(\varepsilon) \lim _{t \rightarrow \infty} \frac{\log \# P_{g_{0}}(t \cdot a(\varepsilon))}{t \cdot a(\varepsilon)}=a(\varepsilon) \cdot h_{T}\left(g_{0}\right) .
\end{aligned}
$$

Since $\varepsilon>0$ was arbitrary, it follows that:

$$
h_{T}(g) \leqq h_{T}\left(g_{0}\right) \cdot \int_{(S M)_{g}}\|v\|_{g_{0}} d \mu_{g} .
$$

Replacing $g$ by $g_{0}$ we obtain:

$$
h_{T}\left(g_{0}\right) \leqq h_{T}(g) \cdot \int_{(S M)_{g_{0}}}\|v\|_{g} d \mu_{g_{0}} .
$$

Proof of Theorem 3. Let $g_{\lambda}$ be a $C^{1}$ perturbation of $g=g_{0}$. Then Proposition 8 yields:

$$
\begin{aligned}
h_{T}\left(\phi_{0}\right)\left(\frac{1}{\int_{(S M)_{g_{0}}}\|v\|_{g_{\lambda}} d \mu_{g_{0}}}-1\right) & \leqq h_{T}\left(g_{\lambda}\right)-h_{T}\left(g_{0}\right) \\
& \leqq h_{T}\left(\phi_{0}\right)\left(\int_{(S M)_{g_{\lambda}}}\|v\|_{g_{0}} d \mu_{g_{\lambda}}-1\right) .
\end{aligned}
$$

Linearizing $\|v\|_{g_{\lambda}}$ in $\lambda$ at $\lambda=0$, we have:

$$
\|v\|_{g_{\lambda}}=\|v\|_{g_{0}}+\left.\lambda \cdot \frac{\partial}{\partial s}\right|_{s=0}\|v\|_{g_{s}}+o(\lambda, v) .
$$


Substituting this in the above inequality we obtain for suitable $o_{1}(\lambda), o_{2}(\lambda)$ :

$$
\begin{aligned}
& h_{T}\left(g_{0}\right)\left(-\left.\lambda \int_{(S M)_{q_{0}}} \frac{\partial}{\partial s}\right|_{s=0}\|v\|_{g_{s}} d \mu_{0}+o_{1}(\lambda)\right) \leqq h_{T}\left(g_{\lambda}\right)-h_{T}\left(g_{0}\right) \\
& \quad \leqq h_{T}\left(g_{0}\right)\left(-\left.\lambda \int_{(S M)_{q_{j}}} \frac{\partial}{\partial s}\right|_{s=0}\|v\|_{g_{s}} d \mu_{g_{\lambda}}+o_{2}(\lambda)\right) .
\end{aligned}
$$

The weak continuity of the Margulis measure implies that:

$$
\begin{aligned}
\left.\frac{\partial}{\partial \hat{\lambda}}\right|_{\lambda=\lambda_{0}} h_{T}\left(g_{\lambda}\right) & =-\left.h_{T}\left(g_{0}\right) \cdot \int_{(S M)_{g_{0}}} \frac{\partial}{\partial \hat{\lambda}}\right|_{\lambda=\lambda_{0}}\|v\|_{g}, d \mu_{g_{0}} \\
& =\left.\frac{-h_{T}\left(g_{0}\right)}{2} \cdot \int_{(S M)_{q_{0}}} \frac{\partial}{\partial \hat{\lambda}}\right|_{\lambda=\lambda_{0}} g_{\lambda}(v, v) d \mu_{g_{0}} .
\end{aligned}
$$

Proof of Theorem 4. Let $(M, g)$ be a compact $C^{2}$ Riemannian manifold of negative curvature of arbitrary dimension and let $l_{g_{0}}$ denote the Liouville measure with respect to $g_{0}$. A straightforward calculation [Be] shows:

$$
\left.\int_{(S M)_{q_{0}}} \frac{\partial}{\partial \lambda}\right|_{\lambda=\lambda_{0}} g_{\lambda}(v, v) d l_{g_{0}}=\left.\frac{\omega(n-1)}{n} \frac{\partial}{\partial \lambda}\right|_{\lambda=\lambda_{0}} \operatorname{vol}_{g_{\lambda}}(M),
$$

where $\omega(n-1)$ denotes the volume of the Euclidean $(n-1)$ sphere. The formula for the derivative of the entropy immediately shows that $g_{0}$ is a critical point in $\mathfrak{R}(M)$ if the Margulis measure and the Liouville measure of $g_{0}$ coincide. Now assume that $g_{0}$ is a critical point for $h_{T}: \Re(M) \rightarrow \mathbb{R}$. Let $\xi$ be a symmetric $(0,2)$ tensor on $M$ such that $g_{\lambda}=g_{0}+\lambda \xi$ is a perturbation of $g_{0}$ and $g_{\lambda} \in \mathfrak{R}(M)$. Then:

$$
\left.\frac{\omega(n-1)}{n} \cdot \frac{\partial}{\partial \lambda}\right|_{\lambda=\lambda_{0}} \operatorname{vol}_{g_{\lambda}}(M)=\int_{(S M)_{q_{0}}} \xi(v, v) d l_{g_{0}}=0 .
$$

The formula for the derivative of entropy implies that:

$$
\int_{(S M)_{g_{0}}} \xi(v, v) d \mu_{g_{0}}=0
$$

where $\mu_{g_{0}}$ is the Margulis measure of $g_{0}$. Define the functionals

$$
L_{l_{g_{0}}}(f)=\int_{(S M)_{q_{0}}} f d l_{g_{0}} \text { and } L_{\mu_{g_{0}}}(f)=\int_{(S M)_{g_{0}}} f d \mu_{g_{0}}
$$

where $f$ is a smooth function. Let $\Omega_{0,2}$ be the space of symmetric $(0,2)$ tensors on $M$. If $\xi \in \Omega_{0,2}$, then $\xi(v, v)$ is a smooth function on $S M_{g_{0}}$. The discussion above shows that if $g_{0}$ is a critical point for $h_{T}$, then $\operatorname{Ker} L_{l_{g_{0}} \mid \Omega_{0,2}} \subseteq \operatorname{Ker} L_{\mu_{g_{0}} \mid \Omega_{0,2}}$.

Proof. Choose $r \in \Omega_{0,2}$ and assume $L_{l_{q_{0}}}(r)=a$. Define $q \in \Omega_{0,2}$ as $q=a \cdot g_{0}$. This implies: $L_{l_{g_{0}}}(r-q)=L_{l_{q_{0}}}(r)-a=0$. By hypothesis we obtain:

$$
0=L_{\mu_{q_{0}}}(r-q)=L_{\mu_{g_{0}}}(r)-L_{\mu_{q_{0}}}(q)=L_{\mu_{g_{0}}}(r)-a .
$$

Hence, $L_{\mu_{q_{0}}}(r)=L_{l_{g_{0}}}(r)$.

This lemma shows that $L_{l_{g_{0}}}(f)=L_{\mu_{g_{0}}}(f)$ for all functions $f: S M_{g_{0}} \rightarrow \mathbb{R}$ which arise from symmetric $(0,2)$ tensors on $M$. Let $\pi:(S M)_{g_{0}} \rightarrow M$ denote the canonical 
projection. If we associate to each smooth function $f: M \rightarrow \mathbb{R}$ the symmetric $(0,2)$ tensor $f \cdot g_{0}$, then Lemma 8 implies that $L_{l_{g_{0}}}(f \circ \pi)=L_{\mu_{g_{0}}}(f \circ \pi)$ for all smooth functions $f: M \rightarrow \mathbb{R}$.

The measures $l_{g_{0}}$ and $\mu_{g_{0}}$ are invariant under the geodesic flow $\phi^{t}$ and coincide on $\pi^{-1}(B(M)):=\left\{\pi^{-1}(A) \mid A \in B(M)\right\}$, where $B(M)$ is the $\sigma$-algebra of Borel subsets on $M$. We would like to say this implies that the measures coincide on the $\sigma$-algebra of Borel subsets of $S M$, and that $g_{0}$ is locally symmetric (cf. Conjectures 2 and 3 above). Unfortunately we can only prove this for surfaces.

Lemma 9. Let $\left(M, g_{0}\right)$ be a compact negatively curved surface and suppose that $l_{g_{0}}$ and $\mu_{g_{0}}$ coincide on $\pi^{-1}(B(M))$. Then $g_{0}$ is a metric of constant negative curvature.

Proof. The function $\mathrm{H}^{+}(v)$, which assigns to each $v \in S M_{g_{0}}$ the normal curvature of the unstable horocycle orthogonal to $v$, satisfies the Riccati differential equation $\dot{u}+u^{2}+K\left(\pi \circ \gamma_{v}(t)\right)=0$, where $\gamma_{v}$ is the geodesic with initial velocity $v$ and $K$ is the Gaussian curvature. If we integrate the Riccati equation with respect to $\mu_{g_{0}}$, we obtain:

$$
\int_{(S M)_{g_{0}}}\left(H^{+}\right)^{2} d \mu_{g_{0}}=\int_{(S M)_{g_{0}}}-K(\pi \circ v) d \mu_{g_{0}} .
$$

Since all of our metrics are normalized to have area 1, our hypothesis and the Gauss-Bonnet Formula imply:

$$
\int_{(S M)_{g_{0}}}-K(\pi \circ v) d \mu_{g_{0}}=\int_{(S M)_{g_{0}}}-K(\pi \circ v) d l_{g_{0}}=-2 \pi E,
$$

where $E$ is the Euler characteristic of $M$. The Ruelle entropy estimate [R] and Jensen's inequality imply:

$$
h_{\text {top }}^{2} \leqq\left(\int_{(S M)_{g_{0}}} H^{+} d \mu_{g_{0}}\right)^{2} \leqq \int_{(S M)_{g_{0}}}\left(H^{+}\right)^{2} d \mu_{g_{0}} .
$$

Putting everything together we obtain $h_{\text {top }}^{2} \leqq-2 \pi E$. In Theorem 3.1 of [K2], the first author proves that $h_{\text {top }}^{2} \geqq-2 \pi E$, and the inequality is strict unless $g_{0}$ is a metric of constant negative curvature. We conclude that $g_{0}$ is a metric of constant negative curvature.

\section{Appendix. Proof of Proposition 3}

Proof. Let $\Psi$ be $C^{1}$ close to $\Phi$. The idea is to use the Implicit Function Theorem to solve:

$$
\Psi \circ u=\gamma \cdot D_{\Phi} u \text { for } u \in C_{\Phi}^{\alpha} \text { and } \gamma \in C^{\alpha}(X)
$$

Define:

$$
\begin{aligned}
& G: V^{1}(X) \times C_{\Phi}^{0}(X) \times C^{0}(X, \mathbb{R}) \rightarrow C^{0}(X, T X), \\
& G(\Psi, u, \gamma)=\Psi \circ u-\gamma \cdot D_{\Phi} u,
\end{aligned}
$$

where $V^{1}(X)$ denotes the space of $C^{1}$ vector fields on $X$ and $C^{0}(X, Y)$ the space of $C^{0}$ functions from $X$ to $Y$. The spaces introduced above are Banach manifolds 
and one can easily prove that $G$ is a $C^{1}$ map. It is clear that

$$
G(\Phi, \mathrm{id}, 1)=0 .
$$

Let $D_{2,3} G$ be the derivative with respect to the second and third variables. Then

$$
D_{2,3} G(\Phi, \mathrm{id}, 1): V_{\Phi}^{0}(X) \times C^{0}(X, \mathbb{R}) \rightarrow V^{0}(X)
$$

is given by:

$$
\begin{aligned}
D_{2,3} G(\Phi, \mathrm{id}, 1)(v, \gamma) & =D \Phi(v)-D v(\Phi)-\gamma \cdot \Phi \\
& =[\Phi, v]-\gamma \cdot \Phi \\
& =\left.\frac{d}{d t}\right|_{t=0} D \phi^{-t} v^{\circ} \phi^{t}-\gamma \cdot \Phi,
\end{aligned}
$$

where $V_{\Phi}^{0}(X)$ denotes the subspace of the space of continuous vector fields $V^{0}(X)$ such that the directional derivative with respect to $\Phi$ exists. $\left(T_{\mathrm{id}} C_{\Phi}^{0}=V_{\Phi}^{0}(X)\right)$. Because the kernel of the Lie derivative

$$
L_{\Phi}(v)=[\Phi, v]=\left.\frac{d}{d t}\right|_{t=0} D \phi^{-t} v^{\circ} \phi^{t}
$$

contains the infinitesimal generator $\Phi$, it follows that $D_{2,3} G$ is not invertible. To overcome this problem choose a section transverse to $\Phi$. For instance define $\Phi^{\perp}=\Gamma\left(E^{s}\right) \oplus \Gamma\left(E^{u}\right)$ and $V_{\Phi, \perp}^{0}(X)=V_{\Phi}^{0} \cap \Phi^{\perp}$. Then it is easy to show that $L_{\Phi}^{\perp}: V_{\Phi, \perp}^{0}(X) \rightarrow V_{\Phi, \perp}^{0}(X)$ has an inverse given by:

$$
\begin{aligned}
& \left(L_{\Phi}^{\perp}\right)^{-1}\left(v_{u}\right)=-\int_{0}^{\infty} D \phi^{-s} v_{u} \phi^{s} d s, \quad v_{u} \in \Gamma\left(E^{u}\right), \\
& \left(L_{\Phi}^{\perp}\right)^{-1}\left(v_{s}\right)=\int_{0}^{\infty} D \phi^{x} v_{s} \phi^{-x} d x, \quad v_{s} \in \Gamma\left(E^{s}\right) .
\end{aligned}
$$

This proves that $(v, \gamma) \rightarrow L_{\Phi}^{\perp}(v)-\gamma \cdot \Phi=D_{2,3} G(\Phi, \mathrm{id}, 1)(v, \gamma)$ is invertible. Now apply the Implicit Function Theorem to the equation $G=0$ with $C_{\Phi}^{0}$ replaced by $C_{\Phi, \perp}^{0}(X)=\exp \left(V_{\Phi, \perp}^{0}(X)\right)$.

\section{References}

[A] Anosov, D.: Geodesic Flows on closed Riemannian manifolds with negative curvature. Proc. Stek. Inst. 90 (1967)

[B] Bowen, R.: Periodic orbits for hyperbolic flows. Am. J. Math. 94, 1-30 (1972)

[Be] Besse, A.: Einstein Manifolds. Berlin, Heidelberg, New York: Springer 1987

[BGS] Ballmann, W., Gromov, M., Schroeder, V.: Manifolds of non-positive curvature. Prog. Math., vol. 61. Baset, Boston: Birkhäuser 1985

[BK] Burns, K., Katok, A.: Manifolds with non-positive curvature. Ergod. Th. Dyn. Sys. 5, 307-317 (1985)

[C] Contreras, G.: Regularity of Topological and Metric Entropy of Hyperbolic Flows, preprint

[FM] Freire, A., Mañé, R.: On the entropy of the geodesic flow in manifolds without conjugate points. Invent. Math. 69, 375-392 (1982)

[K1] Katok, A.: Lyapunov exponents, entropy and periodic orbits for diffeomorphisms. Publ. Math. IHES 51, 137-173 (1980) 
[K2] Katok, A.: Entropy and closed geodesics. Ergod. Th. Dyn. Syst 2, 339-367 (1982)

[K3] Katok, A.: Nonuniform hyperbolicity and structure of smooth dynamical systems. Proc. of Intl. Congress of Math. 1983, Warszawa, vol. 2, 1245-1254

[K4] Katok, A.: Four applications of conformal equivalence to geometry and dynamics. Ergod. Th. Dyn. Sys. 8, 139-152 (1988)

[KKPW] Katok, A., Knieper, G., Pollicott, M., Weiss, H.: Differentiability and analyticity of topological entropy for Anosov and geodesic flows. Invent. Math. 98, 581-597 (1989)

[LMM] de la Llave, R., Marco, J., Moriyon, R.: Canonical perturbation theory of Anosov systems and regularity results for Livsic cohomology equation. Ann. Math. 123, 537-611 (1986)

[M] Manning, A.: Topological entropy for geodesic flows. Ann. Math. 110, 567-573 (1979)

[Ma1] Margulis, G.: Applications of ergodic theory to the investigation of manifolds of negative curvature. Funct. Anal. Appl., 3, (4), 335-336 (1969)

[Ma2] Margulis, G.: Certain measures associated with $U$-flows on compact manifolds. Funct. Anal. Appl. 4, (1), 55-67 (1969)

[Mi1] Misiurewicz, M.: On non-continuity of topological entropy. Bull. Acad. Polon. Sci., Ser. Sci. Math. Astro. Phys. 19, (4), 319-320 (1971)

[Mi2] Misiurewicz, M.: Diffeomorphisms without any measure with maximal entropy. Bull. Acad. Polon. Sci., Ser. Sci. Math. Astro. Phys., 21, (10), 903-910 (1973)

[N] Newhouse, S.: Continuity properties of entropy. Ergod. Th. Dyn. Sys. 8, 283-300 (1988)

[R] Ruelle, D.: An inequality for the entropy of differentiable maps. Bol. Soc. Brasil. Math., 9, 83-87 (1978)

[W] Walters, P.: An introduction to ergodic theory. Graduate Texts in Math. vol. 79. Berlin, Heidelberg, New York: Springer 1982

[Y] Yomdin, Y.: Volume growth and entropy. Israel J. Math. 57, 285-300 (1987)

Communicated by J.-P. Eckmann 
